\title{
Improved low-cost, MR-compatible olfactometer to deliver tobacco smoke odor
}

\author{
Steven B. Lowen ${ }^{1} \cdot$ Stacey L. Farmer ${ }^{1} \cdot$ Scott E. Lukas ${ }^{1}$
}

Published online: 19 February 2016

(C) Psychonomic Society, Inc. 2016

\begin{abstract}
We describe a low-cost, MRI-compatible olfactometer that delivers fresh cigarette smoke odor, a challenging odorant to present, as well as other odorants. This new olfactometer retains all of the advantages of an earlier design that was capable of only delivering volatile odors (Lowen \& Lukas, Behavior Research Methods, 38, 307-313, 2006). The new system incorporates a novel switching mechanism that allows it to deliver fresh smoke generated from a burning cigarette during a stimulus presentation paradigm that might be employed in a cue-reactivity experiment. An evaluation study established that the olfactometer reliably delivered smoke to the participants and that tobacco smoke was discriminated from other odorants; there were no adverse reactions to the device.
\end{abstract}

Keywords Olfaction · Odor $\cdot$ fMRI $\cdot$ Cue reactivity $\cdot$ Drug cue $\cdot$ Cigarette $\cdot$ Smoke

\section{Introduction}

Functional magnetic resonance imaging (fMRI) studies of human olfaction were first conducted nearly two decades ago (Yousem et al., 1997). Olfaction is likely an important sense for motivation and emotion, as the first cranial (olfactory) nerve synapses directly on to the limbic region of the central nervous system (c.f., Kadohisa, 2013). Olfactory stimuli are

Steven B. Lowen

lowen@mclean.harvard.edu

1 McLean Imaging Center, McLean Hospital/Harvard Medical School, 115 Mill Street, Belmont, MA 02478, USA extremely salient (Herz et al., 2004), and because the responses to odor cues are nearly instantaneous, it is not feasible to expose individuals to odors outside of the scanner suite and then move them into the bore of the magnet for scanning.

The magnetic resonance (MR) environment imposes significant challenges to olfactometer design, due to severe restrictions on the use of ferromagnetic components, and a number of MRI-compatible olfactometer designs have been developed that address these constraints. Lorig et al. (1999) described a sophisticated MR-compatible olfactometer that delivers precise amounts of odorant. Popp et al. (2004) used a continuous positive airway pressure device (CPAP) to deliver odors to participant in fMRI experiments. Vigouroux et al. (2005) described a method for manually delivering odors synchronized to a participant's respiratory patterns while in an MR environment. This synchronization is vital, greatly improving statistical power to detect activation in olfactory experiments (Wang et al., 2014). Johnson and Sobel (2007) developed an olfactometer with controllable flow, humidity, and temperature that yields known concentrations of odorants at the output. All of these olfactometers employ facemasks with flows generally in excess of $1 \mathrm{~L} / \mathrm{min}$. Lowen and Lukas (2006), in contrast, described an extremely low-cost device for fMRI experiments that delivers multiple odors and uses a nasal cannula instead of a facemask. Others have augmented MR-compatible olfactometer technology to include delivering liquids along with odorants, yielding both taste and smell stimuli (Marciani et al., 2006), and delivering odorants via intravenous administration (Miyanari et al., 2007). Sanganahalli et al. (2009) describe the use of a vacuum line for rapidly scavenging remaining odorant from the manifold during placebo (no odor) presentation.

More recent designs have included more sophisticated features and techniques. Lundström et al. (2010) caution against bubbling air through odorant liquids; passing the air 
stream past the surface of odorants greatly reduces the production of aerosols with concomitant fouling of downstream components. They also employ miniature, nearly silent solenoid valves to reduce auditory cues of olfactometer operation. Sommer et al. (2012) present a low-cost MR-compatible device with complete computer control of odorant timing and flow rate, validated with fMRI. The olfactometer presented in Sezille et al. (2013) has automatic detection of breathing patterns so that timing of odor presentation can be synchronized to the beginning of an inhalation, with automatic calibration based on the participants breathing pattern. Airflow to the participant is maintained at a constant level through all phases of operation. Participant responses, control signals, and olfactometer state are stored in a common log file for later processing. The device was validated with behavioral responses, direct reading of gas concentration, and fMRI. Andrieu et al. (2014) present a device that also synchronizes with breathing and handles lower-level commands automatically, with all actions sent to the host computer in a log file. This device was validated for timing using ultraviolet absorption, for concentration using gas chromatography, and for neurological response using fMRI. Another innovative approach employs pinch valves and miniature valve terminal assemblies located close to the participant, both reducing transition times and device footprint (Bestget et al., 2016). Finally, Vedaei et al. (2013) present a review of issues involved in conducting fMRI studies of olfaction. These authors address expected response times and brain locations; issues of habituation, adaptation, concentration, and number of odor presentations; and applications to diseases such as Alzheimer's disease, Parkinson's disease, schizophrenia, and clinical depression. The results of some 45 neuroimaging studies were synthesized in a meta-analysis, yielding a map of brain regions activated by odor (Seubert et al., 2013).

Because of the direct connections between the olfactory system and limbic structures, odors play an important role in cue-induced craving (Schneider et al., 2001) that contributes to many elements in drug and alcohol abuse. However, the challenge is to match the proper odor with the drug, and it is particularly challenging to study tobacco smoke for a number of reasons. Smoke rapidly goes stale, so that it must be generated in real time. It adsorbs to and later desorbs from a wide variety of surfaces, so it must be generated close to research participants and thus must be burned in the bore of the magnet itself. It builds up on solid surfaces, requiring the use of smoke switches with liquid surfaces. And finally, cigarettes selfextinguish at low flow rates, precluding switching by simply turning the air flow on and off. Due to these challenges, none of the previously developed devices can deliver fresh cigarette smoke to human subjects. Finally, many of the previous designs do not include sufficient details in the paper to permit others to construct them. In the present paper we have filled this void by providing detailed instructions on the design and construction of a low-cost olfactometer that has a number of key features that will permit researchers to deliver fresh smoke to research participants.

\section{Design}

Our design goals for the olfactometer were to be able to deliver fresh tobacco smoke and have rapid switching times while maintaining the advantages of an earlier model (Lowen \& Lukas, 2006). The current design uses dedicated air paths for each odorant. It thus avoids the switching delays and extended transients of that previous model (Lowen \& Lukas, 2006), which used only one path from the main apparatus to the participant, in an attempt to reduce complexity and cost (see Appendix 1 for a complete parts list of the present design). A picture of the finished olfactometer appears in Fig. 1. As in Lowen and Lukas (2006), we employ liquid-based odorants and deliver odors to participants via a nasal cannula.

\section{Operation}

A schematic diagram of the olfactometer appears in Fig. 2, and Fig. 3 presents the associated electrical circuit. We use an aquarium pump rated for $4.6 \mathrm{~L} / \mathrm{min}$ into a $60-\mathrm{cm}$ water column to supply a total flow of $0.8 \mathrm{~L} / \mathrm{min}$ to the rest of the

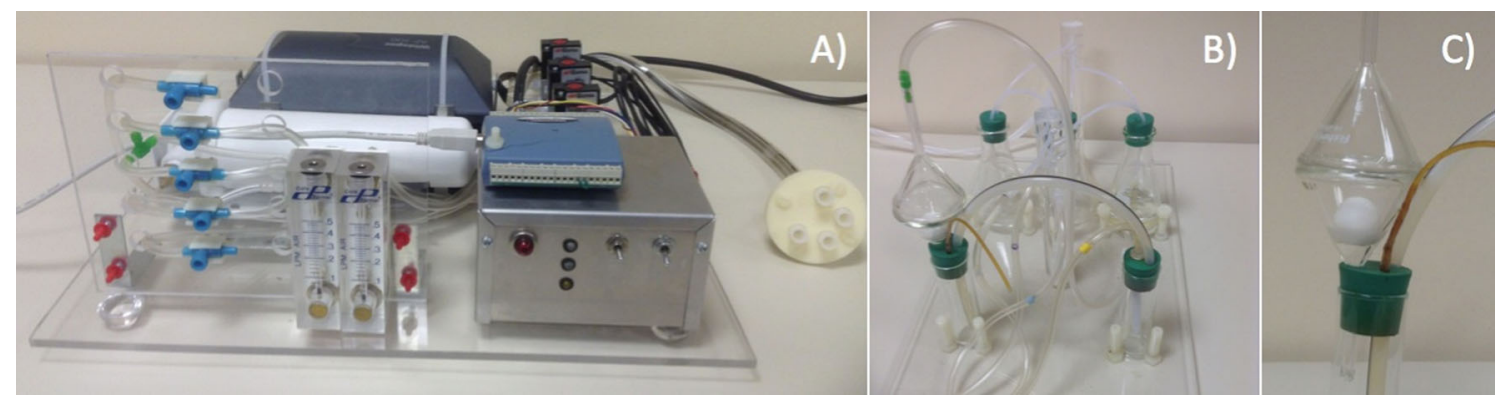

Fig. 1 Photographs of the olfactometer. (A) Base unit, (B) odorant assembly, (C) enlargement of smoke switch 

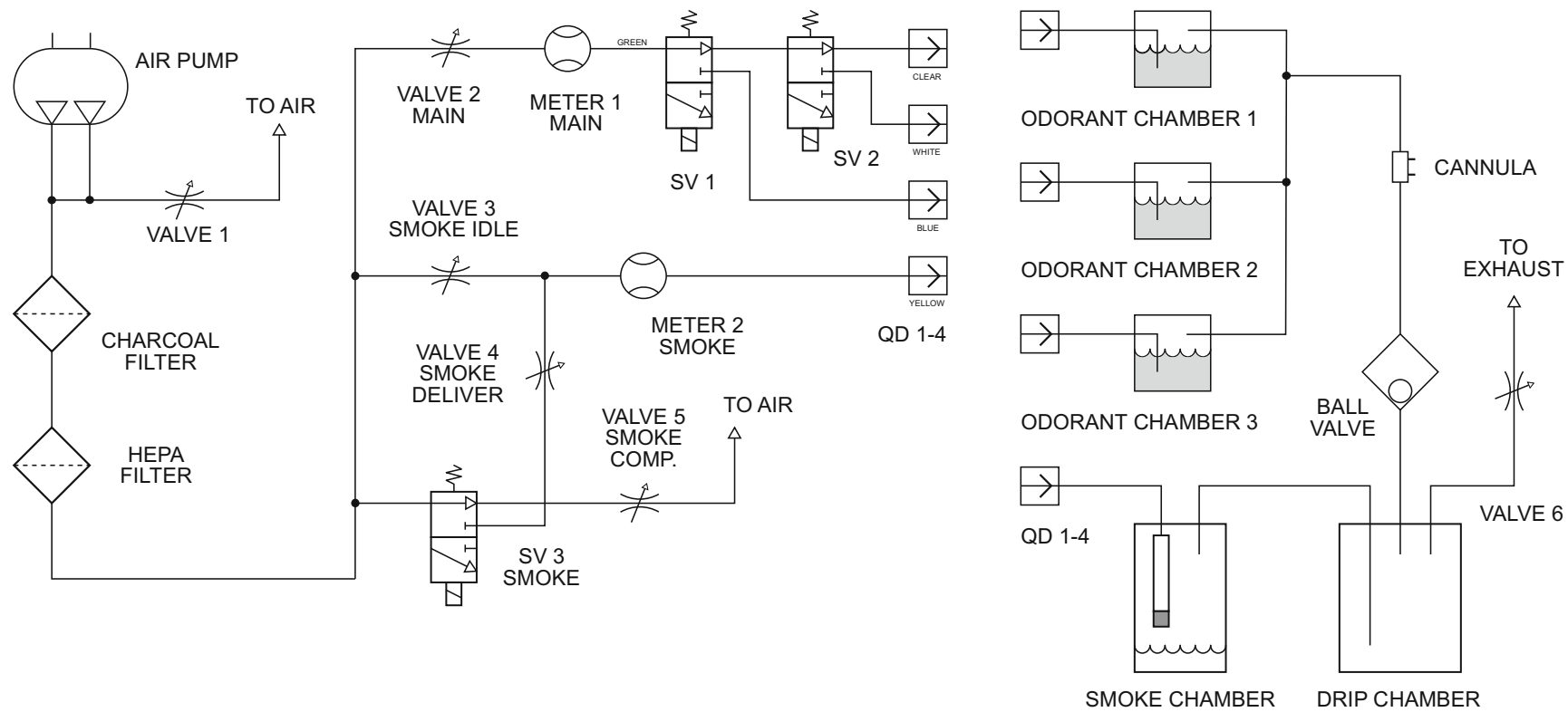

Fig. 2 Schematic representation of the olfactometer airflow. (Left) Base unit, (right) odorant assembly. $S V$ solenoid valve, $Q D$ quick disconnect

olfactometer. As in Lowen and Lukas (2006), an activated charcoal filter removes spurious odors, and a high-efficiency particle-arresting (HEPA) filter blocks any charcoal dust.

Five valves on the base unit set the airflow rates, three solenoid valves control the airflow, and two meters measure it. Airflow passes through quick-disconnects along 15-m (50$\mathrm{ft}$ ) paths towards the odorant assembly that lies in the bore of the magnet, and paths converge to a single line that connects to one side of a nasal cannula. As in Lowen and Lukas (2006), polytetrafluoroethylene (PTFE) and glass are used extensively in components at or downstream of the odorants. The operation of the liquid (non-smoke) odorant pathway follows that of Lowen and Lukas (2006), except that the odor paths joint near the research participant rather than in the base unit.

The other main pathway concerns the smoke delivery system. As cigarettes self-extinguish at flow rates below about $0.25 \mathrm{~L} / \mathrm{min}$, a constant flow of $0.3 \mathrm{~L} / \mathrm{min}$ is provided at all times, increasing to $0.5 \mathrm{~L} / \mathrm{min}$ during smoke delivery; valve \#5 is set to eliminate pressure fluctuations in the rest of the unit when smoke delivery is turned on and off. The smoke airflow passes through the lit cigarette in the smoke chamber. A few $\mathrm{mm}$ of water at the bottom enhances safety. Smoke exits the chamber through 6.35-mm ID PTFE tubing and passes into the smoke switch chamber. This chamber has two outflows: an adjustable valve (\#6; about $15 \mathrm{~cm}$ of cannula tubing) to a vented exhaust system, and the smoke switch itself. The smoke switch comprises two small funnels with open ends taped together, containing a PTFE ball, and sealed with two drops of water on the ball. The far end of the top funnel is connected to 4-mm Tygon tubing, in turn attached to the other side of the cannula. When the air flow is at $0.3 \mathrm{~L} / \mathrm{min}$, the back pressure generated by valve \#6 is not sufficient to break the water seal and raise the PTFE ball, and all smoke exits through the exhaust. At $0.5 \mathrm{~L} / \mathrm{min}$, the increased backpressure lifts the
Fig. 3 Electrical circuit diagrams for the olfactometer. (Left) Line connections and power supply, (right) solenoid valve control (one of three shown). MOV metal oxide varistor, $D B 9$ Dsubminiature nine-pin connector
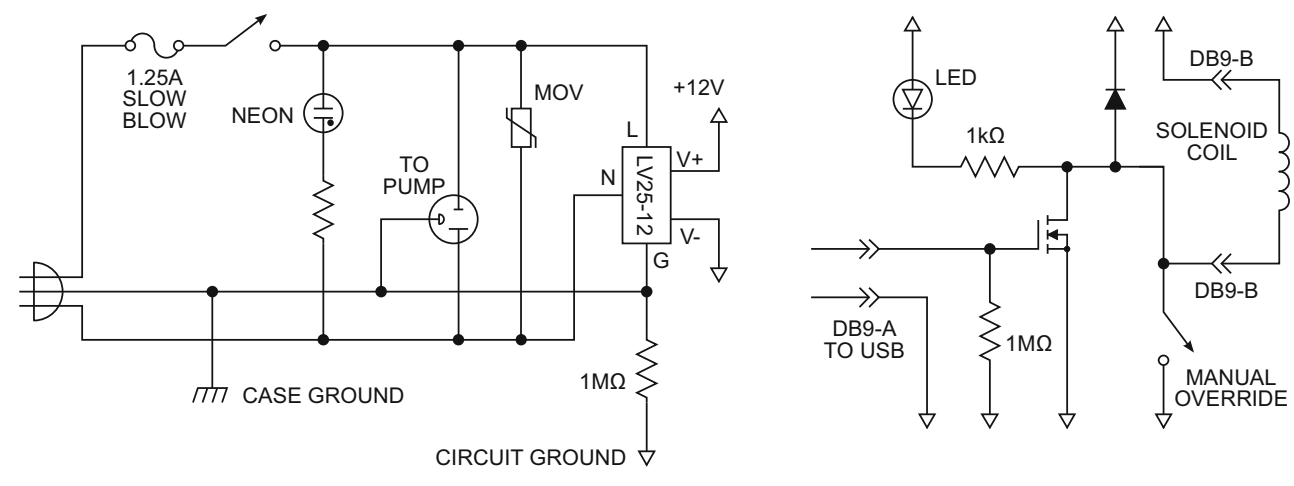
ball and some smoke proceeds through the funnels towards the participant. The flow rate is roughly $0.03 \mathrm{~L} / \mathrm{min}$ (depending on valve \#6), which is sufficient to deliver an easily perceptible smoke odor without being unpleasant. The two chambers are separate to prevent clogging of the lower funnel stem due to water condensing from the smoke.

\section{Construction}

Tubing preparation To eliminate residual odor in new Tygon tubing, we attached it to an inexpensive aquarium pump rated at $2.5 \mathrm{~L} / \mathrm{min}$ and ran it for $50+\mathrm{h}$. The cannula as purchased has two lines that exit the nose attachment and join into a single line ending in a Luer connection. We cut the tubing just before the join, leaving two inputs to the nose attachment. The tubing that was removed, cut into a length of about $15 \mathrm{~cm}$, forms valve \#6.

Stoppers Stoppers were not available with custom sizes and configurations. We chilled the stoppers with dry ice, insulated a drill press, and drilled them before they warmed significantly, chilling the stoppers again between holes. For our design we used a 23/64-in bit for the 10-mm OD glass tubing, 9/32-in for the 8-mm OD PTFE tubing, 7/32-in for the glass funnel, and 1/16-in for valve \#6. The holes enlarge as the stopper returns to room temperature, so the bit sizes are slightly smaller than the desired hole size.

Manifold We connected the three non-smoke odor lines using wye connectors attached to short segments of Tygon tubing, and 1.6-mm reducing connectors at either end. This results in essentially complete switching in less than $1 \mathrm{~s}$ (See Appendix 2), much less than the 2- to 3-s TR used in associated functional MRI experiments, and sufficient for our use.

Electronics We used a Measurement Computing USB1024HLS to interface between USB and the olfactometer; a USB-1024LS can be used in its place.

\section{Evaluation}

To test the performance of the olfactometer device, we conducted the following study.

\section{Method}

Participants Fifteen healthy volunteers, seven females and eight males who ranged in age from 21-36 years (mean \pm S.D. $27 \pm 5$ years) participated. Ages did not differ significantly between the two sexes (two-tailed t-test $\mathrm{p}>0.99$ ).
Participants followed the demographics of the local population (two Asian, four African American, and nine White; one Hispanic and 14 non-Hispanic). The McLean Hospital Institutional Board reviewed and approved the protocol and informed consent used in the study; all participants read and signed the informed consent form.

Experimental design The participants rested comfortably in a soundproofed test chamber during the study with a "white noise" sound generator operating in the room. A disposable nasal cannula was placed in each participant's nostrils and the other end was connected to the olfactometer. The base unit of the olfactometer was positioned outside the chamber, while the odorant assembly (including the smoke apparatus) was placed inside the chamber. A ventilation system removed unused cigarette smoke coming out the exhaust port, and served to remove stray odors from the room. The participants were continuously monitored by video and audio equipment during the experiments. Instructions were presented to the participants via a computer monitor, with responses conveyed using a computer mouse. Immediately before each run of the experiment began, a staff member entered the chamber to light the cigarette and inform the participant that the run was about to begin. Proper functioning of the olfactometer was assessed during and between runs, especially that of the smoke assembly, and runs repeated when indicated (participants bumped into the apparatus, etc.). Five runs were repeated, all for different participants. All participants were informed of the experimental protocol, including the nature of the odors and that one of the trials in each run would have no odor. Only the order of odor presentation remained blind to the participants.

The experiment comprised four runs, each comprising one presentation of artificial rose scent (phenethyl alcohol), acetic acid (white vinegar mixed with an equal quantity of water, yielding $2.5 \%$ acetic acid), and placebo (tap water; no odor), all delivered at a flow rate of $0.3 \mathrm{~L} / \mathrm{min}$; and cigarette smoke (Carlton $100 \mathrm{~s}$ non-menthol with $0.1 \mathrm{mg} /$ cigarette), delivered at approximately $0.03 \mathrm{~L} / \mathrm{min}$. Immediately before the first run, the smoke assembly was primed by burning a cigarette in the assembly at the higher flow rate, so that smoke filled the smoke switch and downstream tubing. The four runs differed only in the order of the four odors, which were counterbalanced for position within the run and with respect to one another; the order was the same for all participants. The order of odor presentation is shown in Table 1. The olfactometer was controlled by a custom-written Presentation program (www.neuro-bs.com).

Each odor presentation event began with a 24-s "time out" to allow the participant's olfactory system to recover (Popp et al., 2004), during which placebo was presented. Following that, participants were instructed to click the right mouse button when they first detected an odor. Next followed a random wait time from 3-27 s (uniformly distributed, mean $15 \mathrm{~s}$ ), after 
Table 1 Order of odor presentations by run

\begin{tabular}{lllll}
\hline Run & Presented & & & \\
\cline { 2 - 5 } & 1 st & 2nd & 3rd & 4th \\
\hline 1 & Rose & Smoke & Vinegar & None \\
2 & Smoke & None & Rose & Vinegar \\
3 & Vinegar & Rose & None & Smoke \\
4 & None & Vinegar & Smoke & Rose \\
\hline
\end{tabular}

which the solenoid was activated and odor flowed into the tubing (or nothing occurred in the case of placebo odor). If participants clicked the right mouse button, a choice of odors was presented (rose, smoke, vinegar, or no odor), and they scrolled through them with the right mouse button. When they had scrolled to the odor they perceived, they clicked the left mouse button to store their choice. Confirming the "no odor" choice returned them to the original screen, before they reported detection, in case they clicked the right mouse button in error; the cancelled detection event was not considered further. Clicking any choice besides "no odor" completed the odor event. All odors were turned off (back to placebo) after $30 \mathrm{~s}$ or identification, whichever occurred first. The detection time (ignoring those that were followed by "no odor"), the identification time, and the odor identified were stored.

Following the odor experiment, the participants completed a short visual analogue scale questionnaire to assess their comfort during the study; questions are listed in Table 2.

\section{Results}

All participants completed the entire experiment, which lasted between 25 and 39 min (mean 30:37, S.D. 3:50). Participants did not find the experiments unpleasant (see Table 2). None of the participants detected any clicks or similar noises during the experiment.

Participants were able to correctly identify different odors better than expected from random chance responding. Table 3 displays a matrix of odor identifications out of 240 trials with four choices. Combining incorrect identifications with misses yielded 156 correct odor identifications out of 240 trials with four choices. Pearson's chi-square test for goodness-of-fit yields an overall probability of $p<10^{-6}$. A similar approach for individual odors yields $p<10^{-6}$ for none, $p<0.05$ for rose, $p<10^{-6}$ for smoke, and $p<10^{-6}$ for vinegar. Based upon a repeated measures ANOVA analysis of individual trial identification data, odor identification accuracy was not contingent on trial number or odor history. Ignoring placebo trials and placebo responses, odors showed sensitivities of $68-83 \%$ and specificities of 85-90\%. Including placebo yielded ranges of $47-83 \%$ and $84-91 \%$. The most likely error was a miss on a phenethyl alcohol odor trial, suggesting that this odorant was weakest.

The latency of odor detection was measured in seconds and analyzed with repeated measures analysis of variance for the three active odors studied. The latencies to detection of individual odors (rose, smoke, vinegar) were significantly different between odors $\mathrm{F}[0,2]=17.535, p<0.001$. Tukey's HSD post hoc analysis revealed that the detection latencies for each odor were all significantly different from each other. The detection time latency for smoke was on average the shortest latency, while rose had the longest average latency to detection (Table 4). Age and sex were not statistically significant covariates for odor detection latencies in our sample of 15 participants. As expected, detection times associated with correct identifications were significantly faster than for incorrect ones (two-tailed t-test $\mathrm{p}=0.037$ ).

\section{Discussion}

At present, there are no published designs of devices that provide fresh cigarette smoke to participants while in an MR environment. We confirmed the overall goal of evaluating the function of the olfactometer to deliver three odors in random order, including tobacco smoke. Participants' identification accuracies are comparable with those of our previous work (Lowen \& Lukas, 2006), using mostly different odorants and a different paradigm (no placebo odors). Average
Table 2 Participant ratings of the experiment, anchored at zero for a completely negative experience, and 100 for a completely positive one. For question 2, odors that were neither pleasant nor unpleasant would correspond to a rating of 50

\begin{tabular}{ll}
\hline Question & Response (mean \pm S.D.) \\
\hline How would you rate your comfort during the experiment? & $81 \pm 17$ \\
How pleasant or unpleasant were the odors? & $46 \pm 20$ \\
Did you experience any nasal irritation from the odors? & $88 \pm 18$ \\
Did you experience any nasal irritation from the cannula? & $96 \pm 5$ \\
How was the duration of the experiment? & $90 \pm 11$ \\
How was the experience overall? & $75 \pm 15$
\end{tabular}


Table 3 Frequencies of Odor Identification Versus Presented Odor The four detected values in each row sum to 60 by design

\begin{tabular}{|c|c|c|c|c|c|c|c|c|}
\hline \multirow[t]{2}{*}{ Presented } & \multicolumn{4}{|c|}{ Detected } & \multicolumn{4}{|c|}{ Specificity and Sensitivity } \\
\hline & None & Rose & Smoke & Vinegar & Sens. 3 & Spec. 3 & Sens. 4 & Spec. 4 \\
\hline None & 40 & 7 & 11 & 2 & - & - & $67 \%$ & $84 \%$ \\
\hline Rose & 24 & 28 & 5 & 3 & $77 \%$ & $90 \%$ & $47 \%$ & $89 \%$ \\
\hline Smoke & 4 & 7 & 38 & 11 & $68 \%$ & $90 \%$ & $63 \%$ & $88 \%$ \\
\hline Vinegar & 0 & 5 & 5 & 50 & $83 \%$ & $85 \%$ & $83 \%$ & $91 \%$ \\
\hline TOTAL & 68 & 47 & 59 & 66 & & & & \\
\hline
\end{tabular}

Sens. sensitivity, Spec. specificity, 3 over active odors only (ignoring placebo trials and responses), 4 over all possibilities (including placebo)

sensitivity and specificity for the present study were $70 \%$ and $88 \%$, respectively, and for Lowen and Lukas (2006) $77 \%$ and $90 \%$, respectively. Much of the difference can be attributed to the use of phenethyl alcohol in the present study, which accounted for $86 \%$ of misses, having a relatively weak odor compared with the other odorants. Although Albrecht et al. (2009) delivered nicotine vapor from nicotine-containing filter paper in nitrogen in order to deliver a single nicotine odorant to participants in an fMRI study, the present study is the first to deliver a smoke odorant. However, the somewhat low sensitivity to smoke odor (63-68\%) was disappointing, suggesting that a higher flow rate for smoke might be useful. That the specificity is relatively high (88-90\%) suggests that smoke odor is distinct and not readily mistaken for other odors.

Tobacco use is a severe public health burden, and the single largest preventable cause of death and disease in the USA (Centers for Disease Control and Prevention, 2015). Many smokers would like to quit, but suffer relapse, and those who do remain abstinent make an average of 8-11 quit attempts (Centers for Disease Control and Prevention, 2013) before achieving long-term success. Relapse prevention is crucial in quit success and thus studies that examine how cues affect current and former smokers will have a greater impact on the development of improved treatment strategies and outcomes.

Because the olfactory nerve has a direct connection to the limbic system (Kadohisa, 2013), odors are potent cues that evoke strong emotional responses (Buck \& Axel, 1991), which makes them very important in cue-induced craving

Table 4 Latencies to odor detection

\begin{tabular}{lcc}
\hline Odor & \multicolumn{2}{l}{ Time (sec; mean \pm S.D. $)$} \\
\cline { 2 - 3 } & Correct ID & Incorrect ID \\
\hline Rose & $13.18 \pm 6.7$ & $14.70 \pm 11.51$ \\
Smoke & $5.68 \pm 4.08$ & $11.81 \pm 4.68$ \\
Vinegar & $8.20 \pm 4.23$ & $4.90 \pm 3.26$ \\
\hline
\end{tabular}

ID identification
(Schneider et al., 2001). MRI modalities have been used to explore a wide range of tobacco-related effects, but despite this, smoking studies rarely employ olfactory cues. In fact, a PubMed search yielded only five out of 217 citations on olfactory cue and none included MR approaches. Being able to study smoking cues in an MR environment offers a uniquely new opportunity to move the field forward and increase our understanding of the neurobiology of smoking behavior that now includes real time brain reactivity to smoke exposure.

\section{Limitations}

While the device performed as originally designed, a number of enhancements would improve its usefulness in a broader range of studies: (1) The Tygon tubing we employed balloons slightly, leading to small delays in odor transitions. Using stiff tubing such as polypropylene will greatly reduce this effect. (2) Although a minor issue, odor adsorption and subsequent desorption at the output is possible. This can be reduced by attaching the 1.6-mm ID PTFE tubing directly (McMasterCarr 2974 K391, for example), although adsorption in the cannula is unavoidable. Employing a vacuum line during placebo conditions, as in Sezille et al. (2013), would help in this regard, and would also decrease the transition times due to dead space. (3) When an odorant is switched on after being off for a long time, the air is saturated with odorant; as the odorant continues to be administered, the concentration decays. Employing odorants with lower boiling points and in particular decreasing flow rates will reduce this effect further, if necessary. (4) The olfactometer as shown delivers only four odors including smoke. However, it is easy to increase the number of odorants, without an excessive increase in cost. (5) The device does not measure participants' breathing as do Sezille et al. (2013) and Andrieu et al. (2014), and so odor administration cannot be synchronized with inspiration without employing other methods of breathing cycle detection. (6) The flow rate cannot be changed during experiments, as in Sommer et al. (2012). 


\section{Conclusion}

We provide detailed instructions for the construction, use, and evaluation of a reliable, inexpensive, MRI-compatible olfactometer that can deliver fresh cigarette smoke as well as other odors to participants in the MR scanner.

Author Note All authors critically reviewed the manuscript and approved of its contents. This work was supported by grant R21DA027062 to SBL, and no authors report any conflicts of interest. As a disclaimer, and like Johnson and Sobel (2007), the design presented here derives from our work, and was not influenced by any interaction with the manufacturers or suppliers of the parts we employed.

\section{Appendix 1}

\section{Parts list}

We present here a complete listing of parts and sources. We purchased some of the less critical parts at local hardware stores rather than at the sources listed below, but either should suffice. Many parts can be substituted. The parts were selected for functionality and low cost. We have no financial relationship with any of the manufacturers or suppliers listed below. Our total bill for materials was less than US $\$ 1,100$ (all monetary values are in US dollars).

\section{Air path}

Pump: Tetra whisper AP300 aquarium air pump

$\$ 41.49$ at www.amazon.com

Tygon tubing: 5554 K47 5/32" ID, 7/32" OD

$\$ 33$ for $50 \mathrm{ft}(\times 5)$ at www.mcmaster-carr.com

Valves and tee connectors: Airline control kit

\$3.05 at www.amazon.com

Charcoal filter: Whirlpool WHCF-IMTOS

$\$ 10.99$ at www.amazon.com

Associated fitting: Cole-Parmer EW-31701-08

$\$ 3.75$ for a package of 10 at www.coleparmer.com

High-efficiency particle arresting (HEPA) filter: Tiara Medical Systems TBF-200S

This company no longer exists, but the "In-Line Sanitary Filter", a similar device, is available for $\$ 4.50$ at www. northernbrewer.com

Flow meter: Cole-Parmer EW-32460-40

$\$ 64.00(\times 2)$ at www.coleparmer.com

Associated fitting: Cole-Parmer EW-30704-08

$\$ 25.00$ for a package of $10(\times 2)$ at www.coleparmer.com

Solenoid valve: Ingersoll-Rand/ARO P251SS-012-D

$\$ 34.30(\times 3)$ at www.grainger.com

Reducing connectors: EW-30703-43

$\$ 15$ for a package of 10 at www.coleparmer.com PTFE 3/4" ball: $9660 \mathrm{~K} 28$
\$2.31 at www.mcmaster.com

PTFE 1/4" ID, 5/16" OD tubing: 53935 K261

$\$ 7.04$ for $2 \mathrm{ft}$ at www.mcmaster.com

Vials: 03-339-26H $29 \mathrm{~mm} \times 94 \mathrm{~mm}$ glass vial

$\$ 142.40$ for a package of 144 at www.fishersci.com

Flasks: S00143 $125 \mathrm{ml}$ Erlenmeyer

$\$ 43.50$ for a package of 12 at www.fishersci.com

Stoppers for flasks: EW-62991-16 neoprene, size 5 1/2

$\$ 39$ for a package of 23 at www.coleparmer.com

Stoppers for vials: EW-62991-14 neoprene, size 5

$\$ 38$ for a package of 23 at www.coleparmer.com

Funnels: FB-601-5845 glass

$\$ 53.90$ for a package of 6 at www.fishersci.com

Y connectors for Tygon tubing: 2974 K283

$\$ 13.16$ for a package of 10 at www.memaster.com

Cannula: Disposable Adult (4000 F)

$\$ 15.15$ each at $\mathrm{http}: / /$ store.invivocorp.com

Cigarette holder: S37608G glass tubing 10-mm OD 8-mm ID

\$57.15 at www.fishersci.com

Quick disconnects: EW-06290-05

$\$ 28.25$ for a package of 20 at www.coleparmer.com

PTFE 1/16" ID, 1/8" OD tubing: 5239 K24

$\$ 50$ for $50 \mathrm{ft}$ at www.mcmaster.com

\section{Electrical}

Mosfet: we used FDS6982S, but that part is no longer available. A similar device is FDS8949

$\$ 0.659(\times 3)$ at www.newark.com

Power supply: TDK Lambda - LS25-12, 12 V @ 2.1A

$\$ 16.50$ at www.newark.com

Power entry connector: 92 N4145

\$2.27 at www.newark.com

Manual override switch (other): 69 K7015 on-none-on

\$5.98 at www.newark.com

Manual override switch (smoke): 98 K6231 SPDT

$\$ 1.75$ at www.newark.com

Fuse: 48 K9575 1.25A time-delay

$\$ 0.537$ at www.newark.com

Varistor: 58 K7216

$\$ 0.19$ at www.newark.com

Indicator lamp: 16 F736

$\$ 6.49$ at www.newark.com

Power entry connector: 17B7286

$\$ 6.43$ at www.newark.com

Power cord: 98 K6026

$\$ 6.36$ at www.newark.com

Metal enclosure: 83 F8927

$\$ 10.07$ at www.newark.com

Diode: 58 K9605 1 N5401 3A $100 \mathrm{~V}$

$\$ 0.30(\times 3)$ at www.newark.com

Gate and ground resistors: 71 M0986 1M $\Omega$ 1/2 W $5 \%$ 
\$0.025 (×4) at www.newark.com

LED resistors: $74 \mathrm{M} 32971 \mathrm{k} \Omega$ 1/2 W $5 \%$

$\$ 0.034(\times 3)$ at www.newark.com

DB9 connector plug: 01 M1411

$\$ 6.97$ at www.newark.com

DB9 connector socket: 79 K4993

$\$ 1.97$ at www.newark.com

\section{Appendix 2}

Power-law decay of odorant concentrations

Using a tube of radius $\mathrm{R}$ and length $\mathrm{L}$ with odorant flowing through it at an average velocity $\mathrm{v}_{\mathrm{a}}$, and assuming laminar flow, the flow velocity $\mathrm{v}$ at a radius $\mathrm{r} \leq \mathrm{R}$ is given by

$\mathrm{v}(\mathrm{r})=2 \mathrm{v}_{\mathrm{a}}\left[1-(\mathrm{r} / \mathrm{R})^{2}\right]$

(Kay, 1985). Suppose the flow entering the tubing changes to air at time $\mathrm{t}=0$. For a participant at the other end of the tubing, the odorant will change to air gradually, given the differing flow velocities at different radii. At a radius $r$, the flow will change type at a time $t=L / v(r)$. Solving for $r$ yields $\mathrm{r}=\mathrm{r}_{0} \equiv \mathrm{R}\left(1-\mathrm{t} / \mathrm{t}_{0}\right)^{1 / 2}$ for $\mathrm{t}>\mathrm{t}_{0}$, where $\mathrm{t}_{0} \equiv \mathrm{L} /\left(2 \mathrm{v}_{\mathrm{a}}\right)$. For earlier times, no air has reached the participant, and so odorant flow has not changed. For larger radii, the flow still contains odorant. To obtain the total odorant flow $\mathrm{Q}$ remaining, we include the cross-sectional area at a radius $\mathrm{r}$, namely $2 \pi \mathrm{r} \mathrm{dr}$, and integrate:

$\mathrm{Q}(\mathrm{t})=\int 2 \mathrm{v}_{\mathrm{a}}\left[1-(\mathrm{r} / \mathrm{R})^{2}\right] 2 \pi \mathrm{r} \mathrm{dr}=\pi \mathrm{r}^{2} \mathrm{v}_{\mathrm{a}}\left(\mathrm{t}_{0} / \mathrm{t}\right)^{2}=\mathrm{Q}(0)\left(\mathrm{t}_{0} / \mathrm{t}\right)^{2}$,

where the limits of integration are $r_{0}$ and $\mathrm{R}$. We note that the tubing has a volume $\mathrm{V}=\pi \mathrm{r}^{2} \mathrm{~L}$, so $(1 / 2) \mathrm{V} / \mathrm{Q}(0)=(1 / 2)\left(\pi \mathrm{r}^{2}\right.$ $\mathrm{L}) /\left(\pi r^{2} v_{a}\right)=(1 / 2) L / v_{a}=t_{0}$, so $t_{0}$ is half the average transit time from the manifold to the participant. For the design of Lowen and Lukas (2006), the average transit time was $6 \mathrm{~s}$, and so reducing odorant concentrations to $1 \%$ of the maximum required $30 \mathrm{~s}$. The current design has an average transit time of approximately $180 \mathrm{~ms}$, and achieves a reduction to $1 \%$ concentration in less than $1 \mathrm{~s}$.

\section{References}

Albrecht, J., Kopietz, R., Linn, J., Sakar, V., Anzinger, A., Schreder, T., . . ., Wiesmann, M., (2009). Activation of olfactory and trigeminal cortical areas following stimulation of the nasal mucosa with low concentrations of S(-)-nicotine vapor-an fMRI study on chemosensory perception. Human Brain Mapping, 30, 699-710.

Andrieu, P., Bonnans, V., Meneses, J., Millot, J., Moulin, T., \& Gharbi, T. (2014). A modular, computer-controlled system for olfactory stimulation in the MRI environment. Behavior Research Methods, 46, $178-184$.
Bestget, A-K., Schulze, P., Kuchinke, L., Suchan, B., Derdak., T., Otto, T., . . ., Sucker., K., (2016). Journal of Neuroscience Methods 261, 85-96.

Buck, L., \& Axel, R. (1991). A novel multigene family may encode odorant receptors: a molecular basis for odor recognition. Cell, 65 , $175-187$.

Centers for Disease Control and Prevention CDC (2015) Cigarette smoking in the United States. Available at: http://www.cdc.gov/ tobacco/campaign/tips/resources/data/cigarette-smoking-in-unitedstates.html. Accessed 3 June 2015.

Centers for Disease Control and Prevention CDC (2013). Tobacco-use cessation. Available at: http://www.cdc.gov/ workplacehealthpromotion/implementation/topics/tobacco-use. html. Accessed 3 June 2015.

Herz, R. S., Eliassen, J., Beland, S., \& Souza, T. (2004). Neuroimaging evidence for the emotional potency of odor-evoked memory. Neuropsychologia, 42, 371-378.

Johnson, B. B., \& Sobel, N. (2007). Methods for building an olfactometer with known concentration outcomes. Journal of Neuroscience Methods, 160, 231-245.

Kay, J. M. (1985). Fluid mechanics and transfer processes. New York: Cambridge University Press.

Kadohisa, M. (2013). Effects of odor on emotion, with implications. Frontiers in System Neuroscience, 7, 66.

Lorig, T. S., Elmes, D. G., Zald, D. H., \& Pardo, J. V. (1999). A computercontrolled olfactometer for fMRI and electrophysiological studies of olfaction. Behavior Research Methods, Instruments, \& Computers, 31, 370-375. (See also an update at http://psych.wlu.edu/cnl/ olfactometer_construction.htm)

Lowen, S. B., \& Lukas, S. E. (2006). A low-cost, MR-compatible olfactometer. Behavior Research Methods, 38, 307-313.

Lundström, J. N., Gordon, A. R., Alden, E. C., Boesveldt, S., \& Albrecht, J. (2010). Methods for building an inexpensive computer-controlled olfactometer for temporally-precise experiments. International Journal of Psychophysiology, 78, 179-189.

Marciani, L., Pfeiffer, J. C., Hort, J., Head, K., \& Bush, D. (2006). Improved methods for fMRI studies of combined taste and aroma stimuli. Journal of Neuroscience Methods, 158, 186-194.

Miyanari, A., Kaneoke, Y., Noguchi, Y., Honda, M., Sadato, N., Sagara, Y., \& Kakigi, R. (2007). Human brain activation in response to olfactory stimulation by intravenous administration of odorants. Neuroscience Letters, 423, 6-11.

Popp, R., Sommer, M., Müller, J., \& Hajak, G. (2004). Olfactometry in fMRI studies: Odor presentation using nasal continuous positive airway pressure. Acta Neurobiologiae Experimentalis, 64, 171-176.

Sanganahalli, B. G., Bailey, C. J., Herman, P., \& Hyder, F. (2009). Tactile and non-tactile sensory paradigms for fMRI and neurophysiologic studies in rodents. Methods in Molecular Biology, 489, 213-242.

Schneider, F., Habel, U., Wagner, M., Franke, P., Salloum, J. B., Shah, N. J., . ., Zilles K., et al. (2001). Subcortical correlates of craving in recently abstinent alcoholic patients. American Journal of Psychiatry, 158, 1075-1083.

Seubert, J., Freiherr, J., Djordjevic, J., \& Lundström, J. N. (2013). Statistical localization of human olfactory cortex. NeuroImage, 66, 333-342.

Sezille, C., Messaoudi, B., Bertrand, A., Joussain, P., Thévenet, M., \& Bensafi, M. (2013). A portable experimental apparatus for human olfactory fMRI experiments. Journal of Neuroscience Methods, 218, 29-38.

Sommer, J. U., Maboshe, W., Griebe, M., Heiser, C., Hörmann, K., Stuck, B. A., \& Hummel, T. (2012). A mobile olfactometer for fMRI-studies. Journal of Neuroscience Methods, 209, 189-194.

Vedaei, F., Fakhri, M., Harirchian, M. H., Firouznia, K., Lotfi, Y., \& Oghabian, M. A. (2013). Methodological considerations in 
conducting an olfactory fMRI study. Behavioural Neurology, 27, 267-276.

Vigouroux, M., Bertrand, B., Farget, V., Plailly, J., \& Royet, J. P. (2005). A stimulation method using odors suitable for PET and fMRI studies with recording of physiological and behavioral signals. Journal of Neuroscience Methods, 142, 35-44.
Wang, J., Sun, X., \& Yang, Q. X. (2014). Methods for olfactory fMRI studies: Implications of respiration. Human Brain Mapping, 35, 3616-3624.

Yousem, D. M., Williams, S. C,. Howard, R. O., Andrew, C., Simmons, A., Allin, M., .. ., Doty, R. L. (1997). Functional MR imaging during odor stimulation: preliminary data. Radiology, 204, 833-838. 